\title{
ORIGINAL RESEARCH \\ Temporal binding: digging into animal minds through time perception
}

\author{
Antonella Tramacere ${ }^{1,2}$ (1) $\cdot$ Colin Allen $^{1,3}$ (1)
}

Received: 12 March 2021 / Accepted: 21 October 2021 / Published online: 28 January 2022

(c) The Author(s) 2022

\begin{abstract}
Temporal binding is the phenomenon in which events related as cause and effect are perceived by humans to be closer in time than they actually are (Haggard et al. in Nat Neurosci 5(4):382-385, 2002, https://doi.org/10.1038/nn827). Despite the fact that temporal binding experiments with humans have relied on verbal instructions, we argue that they are adaptable to nonhuman animals, and that a finding of temporal binding from such experiments would provide evidence of causal reasoning that cannot be reduced to associative learning. Our argument depends on describing and theoretically motivating an intermediate level of representations between the lower levels of associations of sensory features and higher symbolic representations. This intermediate level of representations makes it possible to challenge arguments given by some comparative psychologists that animals lack higher-level abstract and explicit forms of causal reasoning because their cognitive capacities are limited to learning and reasoning at the basic level of perceptual associations. Our multi-level account connects time perception with causal reasoning and provides a philosophically defensible framework for experimental investigations that have not yet been pursued. We describe the structure of some possible experiments and consider the implications that would follow from a positive finding of temporal binding in nonhuman animals. Such a finding would provide evidence of explicit awareness of causal relationships and would warrant attribution of intermediate representations that are more abstract and sophisticated than the associations allowed by the lower level of the two-level account.
\end{abstract}

Keywords Causal cognition - Animal cognition - Time perception · Psychophysics · Abstract reasoning

Antonella Tramacere antonella.tramacere2@unibo.it

1 Department of Philosophy and Communication Studies, University of Bologna, Bologna, Italy

2 Department of Cultural and Linguistic Evolution, Max Planck for the Science of Human History, Jena, Germany

3 Department of History and Philosophy of Science, University of Pittsburgh, Pittsburgh, PA, USA 


\section{Psychophysics of time perception: a new approach in causal cognition}

Explicit causal reasoning is a crucial aspect of human life which allows humans to represent functional and structural relations in the world, to plan and coordinate actions toward anticipating and managing them, and in general to intervene effectively on objects and events. These explicit abilities are typically differentiated from a set of implicit processes that are widely agreed to be shared with nonhuman animals. Implicit processes allow both humans and nonhuman animals to adjust their behavior in ways that track and exploit causal relations in the world even if those relations are not represented by the subject as causal.

Investigation of causal reasoning in humans has incorporated psychophysical measures in recent years due to the discovery of the phenomenon known as temporal binding, which we describe in detail below. The results of these psychophysical investigations are often interpreted in contrast to a Humean psychological framing (e.g. Eagleman \& Holcombe, 2002). For instance, Blakey et al., (2019, p. 1) write:

[T] he relation between time and causation is more complex than the Humean view suggests. Not only does the timing of events influence causal beliefs, but causal beliefs themselves also affect our perception of the timing of events.

In this paper we argue that psychophysical measurement of the subjective timing of cause-effect relations can be extended to experiments involving nonhuman animals, and that this approach has the potential to restructure the debate of the past couple of decades about causal cognition in animals. The examination of causal cognition in a developmental and comparative perspective is ultimately instrumental to deepening our comprehension of how the human mind emerges, how it evolved, and its similarities to and differences from other animal species.

The recent debate among psychologists and philosophers about animal cognition starts with some terms of agreement: the behavior shown by many animal species is more complex than can be explained by the simplest associative models formulated by Hume, as associations among ideas, and by the early behaviorists, as associations among stimuli or between stimuli and responses. Further, most scientists agree that the differences between human and animal behavior cannot be explained by using a sharp and dichotomic contrast between associative and cognitive mechanisms (see also Buckner, 2011).

However, researchers' interpretations vary dramatically when it comes to describing the cognitive resources posited to underpin animals' abilities to behave in ways that exploit causal relations, which we will refer to as "causal behavior". The disagreement lies on whether there is evidence that animals explicitly represent causal relations and draw theory-like inferences to integrate causal information from different sources using such representations. For instance, when reviewing recent experiments on animal causal cognition, Woodward (2021, p.174) writes, "humans integrate causal representations based on geometrical-mechanical cues with causal representations that are relevant to intervention and action. However, there is evidence that human infants as well as many nonhuman animals fail to do this or at least fail to do it as completely and effectively as adult humans do." 
Woodward's last clause hedges his conclusion about the seeming failure of animals to integrate causal representations, but other scholars (Heyes, 2018b; Papineau \& Heyes, 2006; Penn \& Povinelli, 2007; Povinelli \& Penn, 2011) are convinced that no animals have the kind of abstract, allocentric, rational and explicit representations of causal relations attributed to human adults and children (see Gopnik et al., 2004 for evidence of causal reasoning in children). Attempts by comparative psychologists to experimentally demonstrate causal reasoning in animals have until now been taskdriven, involving interactions with experimenter-designed apparatuses or the capacity of animals to make use of tools. Scholars who deny causal understanding to animals claim that the experimental results to date are consistent with a more modest hypothesis; i.e., animals are predisposed to perceive certain clusters of features as more salient than others when selecting among potential actions to achieve specific outcomes (Povinelli \& Penn, 2011).

According to this view, nonhuman animals do not explicitly represent the relevance of properties such as the rigidity of a tool or the presence of invisible mechanisms when choosing their actions. Although they allow that animals are capable of flexibly acquiring causal behaviors, they insist that animals do not go beyond single-causesingle-effect associations among perceivable features of a situation. These scholars acknowledge that both human and non-human animals have cognitive mechanisms that are differentially responsive to covariation and causal relations, but they attribute the human capacity for causal reasoning to proficiency with a symbolic or linguistic level of mental representation possessed by humans alone.

We refer to this position as the two-level view of causal cognition. The two levels referred to by this view are a first order and a higher order of mental representations. The first order concerns associations among perceptual representations, through which animals can treat some causal relations differently from others, but in a way that is limited to observable features of the present situation. In this way, animals may show sensitivity to temporal priority and causal directionality, which are common to causal relations but not to other types of relations between stimuli. In contrast, the higher order concerns the ability to symbolically or linguistically represent theoretical concepts, such as weight or gravity. Only humans use these higher-order mental representations to assist in at least some problem-solving.

Not all researchers agree with the two-level view. Some researchers adopt a multilevel view of causal cognition and argue that the full spectrum of causal behaviors by animals (and preverbal children) cannot be captured by first-order perceptuallylinked associations alone (Seed et al., 2011). According to this view, some kinds of behavior exhibited by animals rely on a higher level of abstraction than is provided by the first-order associations allowed to animals by the two-level theorists. This intermediate nonlinguistic level of representation is theorized to provide a structural template for causal relations which may support the kind of integration of causal information that is taken to be actually or quite possibly a uniquely human capacity (Penn \& Povinelli, 2007). Adopting a multi-level view means theorizing a variety of mental representations employed by animals and pre-verbal children that cannot be reduced to first-order perceptual representations.

In this paper, we propose a psychophysical approach that is novel in studies of animal causal cognition and potentially fruitful for comparative psychology. In Sect. 2 we 
review the current state of the debate about causal cognition in animals. The alternative approach that we propose builds on recent experiments exploring the connections between explicit causal representation and time perception, which we describe in Sect. 3. Initially reported by Haggard et al. (2002) who called it "intentional binding", the temporal binding effect shows that causality and time are mentally connected in humans: If you recognize your action as causing an effect, you experience an illusory compression of the time between the events. Furthermore, this effect generalizes in humans to cases where the action is by another agent and to non-agential causes (Buehner \& Humphreys, 2009; Suzuki et al., 2019).

In Sect. 4, we make an experimental proposal that could be carried out to measure temporal binding in animals, and in Sect. 5 we discuss the implications of the various possible outcomes of this experiment. The results of temporal binding experiments suggest that the same first-order perceptions can be associated in different ways, thus in Sect. 6 we develop in detail the idea that the connection between time and causality can be used to identify and characterize the existence and distribution of an intermediate, structurally abstract, and likely explicit level of causal representation. In Sect. 7, we explain how the psychophysical approach, including variations on the basic experiment outlined in Sect. 4, is capable of stimulating progress in understanding the properties of mental representations of causality and the evolutionary and developmental emergence of human-like causal cognition, as well as the variety and distribution of causal cognition in nonhuman animals.

\section{The ongoing dispute in animal cognition: focus on causal reasoning}

The ongoing dispute between scholars defending multi-level and two-level views of causal cognition is still very vivid. The former argue that at least some animal species are capable of reasoning about at least some aspects of causality (e.g., Seed et al., 2011; Taylor et al., 2007, 2010), while the latter insist that animals operate by relying only on perceptual features, or on what Penn and Povinelli refer to as 'perceptually linked reasoning', i.e., first-order relational reasoning over perceptual properties (Penn \& Povinelli, 2007, 2009; Povinelli \& Penn, 2011). We note here that 'reasoning' by animals is not the disputed term, since it is also used by Povinelli and colleagues; rather, the dispute is about the format of such reasoning, whether targeted at singlecause-single-effect associations or specifically at representations of causal relations.

Virtually every scholar working in the field of animal cognition agrees on the fact that only human beings acquire and use symbolic representations to reason about causality. ${ }^{1}$

Two-level theorists argue that animal causal cognition can be interpreted by only considering first-order relational reasoning whilst human causal cognition requires

\footnotetext{
1 A subject using symbolic representations is able to externally or imaginatively explore a range of counterfactual situations such as interventions (e.g., ban smoking, develop gene therapy for cancer), causal reversals ('cancer causes smoking'), and other rearrangements and recombinations of the basic representational elements. These representational elements may include natural language expressions (e.g., 'Smoking causes cancer'), representations in a formal language (e.g., 'S $\Longrightarrow \mathrm{C}$ '), or diagrammatic representational formats such as directed graphs.
} 
more abstract higher-order reasoning (Povinelli \& Penn, 2011), being identifiable respectively with perceptually-linked representations (present both in animals and in humans) and symbolic representations (present only in humans).

According to two-level theorists, a subject shows possession of an abstract, higherorder reasoning capacity only through explicit use of language or other symbols. It seems then that if it is not symbolic, it cannot be abstract. This argument implies that animals can only reason through perceptually-linked categories, whereas only humans can conceive of a variety of observable events under the structural, role-based relation of functional and physical aspects of reality (Heyes, 2018a; Povinelli \& Henley, 2020).

On the contrary, multi-level theorists maintain that in order to understand the different behaviors of various animal species and the evolutionary trajectory to human cognition, it is necessary to analyse animal behavior on at least two levels or orders of reasoning (McCormack et al., 2011; Seed et al., 2011). Multi-level theorists thus add an intermediate level to the perceptual and the symbolic ones, which is sometimes referred to as "structural" (Seed et al., 2011). The term "structural" refers to the possibility that animals as well as young children are able to reinterpret first-order perceptual cues in terms of their higher-order abstract relations-i.e., relations such as bigger/smaller, same/different, and cause/effect, which can be generalized across contexts and perceptual modalities. The boundary between animal and human cognition is consequently more multifaceted, and its precise description depends on the species under consideration and the task-context.

A number of experiments on tool use in animals have tried to establish whether first-order relational reasoning is both necessary and sufficient to explain animal causal behavior. Many experiments relied on the idea that a fundamental landmark of a cognitive process is skillful means-end behavior suggesting that individuals are able to reason on the basis of the functional characteristics of the objects to execute flexible intentional actions. Many of these experiments have been modeled after Piaget (1954), who tested children with what is known as the string- or cloth-pulling paradigm, in which subjects interested in out-of-reach rewards suspended by a string or cloth understand that the contact between the support and the reward allows them to pull the support to reach the reward.

The basic task has been adapted to different animal species in a vast number of ways to address whether the subject's performance could be generalized to other perceptual situations (for a review see Jacobs \& Osvath, 2015). Other versions of this task have been developed, requiring the subjects to push instead of to pull, or to act on a variety of supports and connecting devices, such as tubes. Researchers have also varied functionally irrelevant features such as the cloth's color, shape, texture, and the shape and size of the gap, in order to test whether the subjects are operating by applying behavioral rules which transcend contingent perceptual features.

Certainly, caution must be exercised when interpreting negative results and species differences, but it seems reasonable to claim that species differ markedly in the number of trials required to solve the tasks. Humans and many of the nonhuman apes tested, as well as some of the monkeys and a few keas performed significantly above chance after less than a dozen trials. In contrast, most of the monkeys and elephants, one bluefronted parrot, and four pigeons required extensive training, sometimes even more than 100 sessions to attain any solution. 
According to two-level theorists, no matter how fast they are in solving the task, all the subjects in these experiments are using perceptually-linked representations to conditionally bind perceivable properties of reality in ways that support differential behavior between causal and noncausal relationships without explicit representation or understanding of the actual causal relations. Subjects employing perceptually linked representations may tend to show more rigid behavior, for example failing to generalize the important causal relations to novel contexts even if sometimes they appear capable of flexible and creative behavior. However, two-level theorists insist, the representation of the causal relationship does not involve any explicit, abstract representation of causality that is available to the subject for reasoning about a wide range of situations.

On the contrary, according to multi-level theorists, at least in some cases, skillful individuals in these experimental paradigms are using intermediate representations of unperceivable (or currently unperceived) properties of reality and generalize their knowledge to different contexts. According to this view, the behavior of some animals does involve an explicit and to some extent abstract representation of the causal relation. A representation of this type would support the organism's ability to use the visual or tactile properties of an object to determine its potential use as a tool in a novel task requiring (for example) some combination of shape and rigidity, or to understand that a light switch is connected to a light even though the wiring between them is not presently visible. Subjects capable of using this type of representation also form first-order associative representations, but can extract causally relevant functional information such as surface continuity, or the solidity of barriers.

Experimental investigation of whether individuals are able to reason about hidden causes demonstrates how diverse the behavior of various animal species can be when it comes to reasoning about unseen perceptual aspects of reality. Corvids have performed above chance in these experiments (Taylor et al., 2012), while chimpanzees of various ages as well as 3-year-old children failed in inferring the existence of currently unperceivable mechanisms which could guide them to a reward. However, 4-5-yearold children were able to solve the problem when the experimenter provided verbal instructions (Civelek et al., 2020). These results are considered by multi-level theorists as evidence for a multifaceted view of causal cognition. Various species may differentially employ various representational resources, from perceptual to more abstractly intermediate to possibly symbolic, depending on the phylogenetic history, the task and the situation (Starzak \& Gray, 2021).

All together, the different interpretations of the various experiments suggest that much of the opposition between these views to attributing causal reasoning about higher-order properties to animals is due to the fact that two-level theorists fail to distinguish between symbolic and intermediate ways to represent causal relationships, thus drawing a sharp distinction between animal and human capacities of causal reasoning.

Researchers like Povinelli and Heyes, who are among the main proponents of the two-level view, argue that it is both sufficient and necessary to explain animal performance through perceptually linked representations. First-order relational reasoning is, according to Povinelli, the only viable, coherent option to explain animal behavior. It is not even a matter of obtaining behavioral evidence, because on Povinelli's view, 
any higher order process of reasoning is simply impossible to prove in subjects who lack language or explicit symbolic representations.

Instead of prematurely foreclosing this issue, we believe that whether animals and young infants alike use perceptually linked or intermediate levels of representation of causal relations is still an open question which is likely to give rise to further experimentation in the future. Our contribution in the rest of this paper is thus aimed at addressing the empirical possibilities for obtaining evidence of this intermediate level and distinguishing it from first-order relational reasoning only utilizing perceptual features.

\section{Temporal binding: connecting time perception with causal reasoning}

Temporal binding is a fascinating phenomenon showing that under specific conditions, subjects perceive the time between two events as shorter than it is objectively. When a subject intentionally executes an action producing effects or attributes a causal relationship to two (nearly) contiguous external events, both the actions and the effects of the two external events are perceived as closer in time than they actually are. We contend that the subjectivity of time perception can be used as a diagnostic tool for identifying intermediate levels of causal representations, establishing convergence and possibly homology between human causal cognition and that of other animal species.

The first experiment on temporal binding labeled the phenomenon intentional binding (Haggard et al., 2002). Participants were asked to execute an intentional action, a keypress producing a tone. This condition was compared to the involuntary action produced by a transcranial magnetic stimulation (TMS) over the motor cortex, inducing an unintentional muscle twitch in the fingers. Both the intentional action and the involuntary muscle twitch were followed by a tone after $250 \mathrm{~ms}$, and participants had to report (in separate blocks) the time of either the first or of the second event (i.e., the tone). When comparing the timing of the action and the tone to baseline, the authors found that only in the case of the intentional action, the perception of the movement initiation was significantly delayed, whereas perception of the tone was brought forward.

The binding effect in humans has been investigated through a number of different techniques. The original experiment of Haggard and colleagues used the Libet clock, which consists of a dot on the screen of an oscilloscope circulating like the hand of a clock, but more rapidly. The authors could ask participants to report the time of the events, i.e., the action or the ensuing tone. Beyond the Libet clock, alternative techniques have served to replicate the main findings of a time compression of action-effects relations. One of these techniques is called stimulus matching, in which participants' task is to launch a stimulus at exactly the same time as a target stimulus, and which has been used both with adults (Buehner, 2012; Buehner \& Humphreys, 2009) and with 4-year-old children (Blakey et al., 2019).

Another technique involves synchrony judgments, for which participants are required to evaluate whether two cues have occurred simultaneously. Participants had to evaluate the time occurrence of a target stimulus in an action or non-action 
condition. They would perceive two cues, one (e.g., a beep) after executing an action (or after a fixation point disappears on a screen), and another temporally independent cue (e.g., a flash). They would then indicate whether the two cues were simultaneous or not. Cravo et al. (2009) have used this technique for both auditory and visual cues, and have found evidence of temporal shift in perception in the action condition, thus replicating the main findings of the action-effect binding through the synchrony judgment task.

The reduction of the temporal interval between the perception of a voluntary action and its effect has been replicated in several studies (see Moore \& Obhi, 2012 for a review), and often interpreted as a correlate of intentional actions. Specifically, it has been argued that when the individual presses a key intentionally, she is deliberately initiating and executing the action expecting a sensory outcome (the tone). This expectation would produce an anticipation of the tone perception, therefore leading the subject to perceive the initiation of the action (the click on the keypress) and the ensuing sensory effect as closer in time compared to when these events objectively occur.

Subsequent studies however have shown that the binding effect does not necessarily depend on the presence of an action, but rather on inferring causal relations (Buehner, 2012; Buehner \& Humphreys, 2009; Suzuki et al., 2019). These studies have investigated the role of causality in the binding effect, and shown that representing an event as the cause of another one is necessary for shortening the perceived time interval (i.e., the duration) between them. Subjects during the experimental sessions infer that an event is about to take place after another one and formulate this prediction in terms of temporal contiguity, that is in terms of the presence of a certain temporal distance between the first and the second event, no matter whether these events are an action and an effect or externally generated events.

Nevertheless, it is possible that action has an additional role in binding perception of events in time. In fact, some studies suggest that binding is enhanced by the presence of an action (Buehner, 2012, 2015). The motor preparation coinciding with the planning phase might shorten the perceived interstimulus interval further. A recent experiment though has found equal temporal binding in action and non-action conditions (Suzuki et al., 2019). In this case however, the authors matched perceptual stimuli temporally and spatially in the action and non-action conditions (they use a virtual hand acting in an ego-centric perspective). In other words, Suzuki et al. (2019) may have created an illusion of action execution. Because this phenomenon can occur without intentional action, the name actually more often used to identify it is temporal binding, and not intentional binding.

Importantly the binding effect does not occur with non-causal associative learning. In fact, no significant changes in time perception occur when the subjects simply experience that two events have occurred together, like in the baseline control conditions of many temporal binding studies. Temporal shift in event perception is triggered by a causal relation, but not by targets that are equally predictable because merely associated with the predictor (Buehner, 2012). In other words, it is not sufficient for an initial stimulus to be followed by a target stimulus - the former must be perceived as a cause of the latter. That is why even a non-intentional mechanical cause results in 
binding. Attributing causal relations with or without action is a necessary component of the binding effect.

Consider the experiment of Buehner (2012). In the intentional condition, the participants bring about the sound of a tone by pressing a button. In the causal-mechanical condition, the button producing the tone is pressed by a machine with a visible causal mechanism. In both conditions, the tone occurs $250 \mathrm{~ms}$ after the button press. In the control condition, a long pitch sound precedes the tone by $250 \mathrm{~ms}$. The three blocks for the three conditions were composed of 40 trials, randomly administered to the subjects. There was therefore plenty of time for the subject to associate the sound of the pitch with the sound of the tone, as predicted by associative learning theory. Nevertheless, the control condition did not significantly result in binding, insofar as the time perception of the two stimuli was unaffected.

Temporal binding is modulated by different factors, which include contiguity, temporal prediction (the expectation of observing an effect at a specific point in time), and predictive control (the expectation of producing an effect). Regarding contiguity, some studies (Haggard et al., 2002; Ruess et al., 2017) found a decrease of temporal binding with less contiguity (e.g., long delay between events). Other studies (Buehner \& Humphreys, 2009; Nolden et al., 2012; Wen et al., 2015) found that binding increases with longer delay, showing that more research is needed to clarify the time window in which this temporal illusion occurs. Regarding predictability of effects, some studies manipulate the occurrence of the action outcome, and show that highly predictable outcomes increase the binding effect (Moore \& Haggard, 2008).

Interestingly, in causal conditions, unpredictable or absent effects do not abolish the shift in perceived timing, demonstrating that the prior confidence in a causal relation overrides variation in both contiguity and contingency (Desantis et al., 2012; Moore et al., 2009). Thus, although the combination of low-level factors is a primitive source of the causal representation, the temporal shift in event perception collectively depends on the emerging higher-level mental construct of causality (Legaspi \& Toyoizumi, 2019), which modulates the way we perceive the occurrence of past and future events.

\section{Temporal binding in nonhuman animals: an experimental proposal}

Temporal binding experiments in humans have shown that inferring a causal relation between events affects the subject's perception of their timing, demonstrating that the subject's assumption or background knowledge changes the way things are perceived in time. It is an experimental question whether also other animal species experience an analogous temporal illusion. Investigating comparatively the relations between causal reasoning and time perception could add new elements to the current debate on animals' causal cognition.

Many psychophysical experiments regarding time perception in humans have used the rotating spot method, such as the Libet clock. However, this method is not suitable for testing perceptual differences of event timing in agents with poor or absent linguistic capacities. Also, observing the clock in order to report the position of the dot is cognitively demanding, and can distract the subject from performing any additional tasks (Engbert et al., 2007). On the contrary, modifications of both the stimulus 
matching and the synchrony judgment tasks could be employed to investigate time perception in comparative psychology.

Here, we make an experimental suggestion for testing the binding effect in animal subjects through a modification of the synchrony judgment task, used by Cravo et al. (2009) in human adults. In the basic experiment (illustrated in Fig. 1), the animal subjects would be trained to discriminate short from long intervals between two cues, such as two tones, T1 and T2 presented shortly after an orienting 'start' signal. When responding to short intervals animals would receive a food reward if they proceed immediately to location $\mathrm{S}$, but not at location $\mathrm{L}$. The reward/non-reward locations would be switched for long interval events.

When the training phase is completed, the subjects would undergo a yoked experimental design, in which each primary participant (experimental group) is paired with the participant of a yoked control. Subjects in the experimental group would now be provided with a key, button, or lever, which is inert until the 'start' signal is presented. After the start signal, the subjects' voluntary press simultaneously releases the first tone (T1) which is followed after a short variable interval by the second tone (T2), as in the training phase. Animals will consequently be rewarded in the location they go to first.

Training

All subjects are exposed to a start signal followed by two tones T1 and T2 at variable intervals, and they learn to expect a food reward at feeder $\mathbf{S}$ if the T1-T2 interval is short or at feeder $\mathbf{L}$ if interval is longer. They receive no reward if they go to the wrong feeder first.

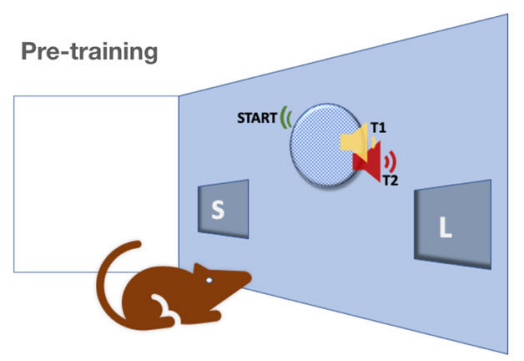

Experiment

Subjects are divided into two groups. Primary animals are provided a lever. After the start signal, a lever press immediately initiates a trial (tone T1 followed by T2 at variable intervals). Yoked (control) animals receive same start-T1-T2 sequence as primary, but not under their own initiation.
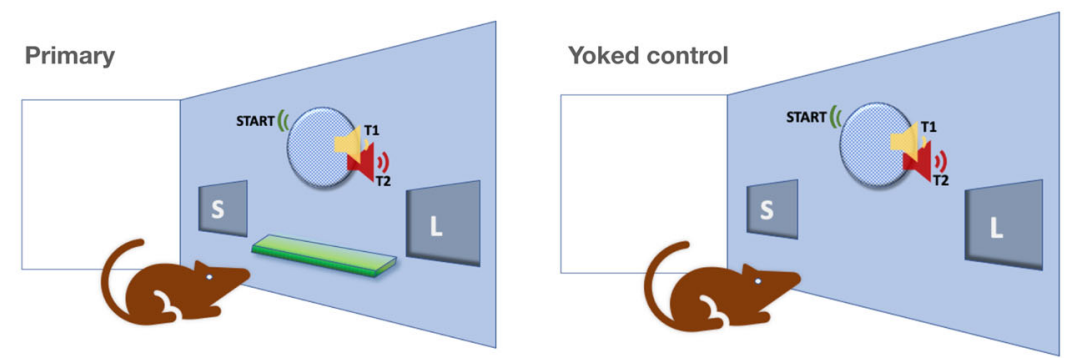

Fig. 1 The basic temporal binding test proposed for animals (the experimental design is illustrated using rats as an example) 
The experimental phase is thus different from the training phase because of the presence of the action: primary animals have now the chance to identify their key press with the ensuing tone. On the other hand, the yoked animals would only experience the start signal followed by the same pairs of stimuli, T1 and T2, separated by variable intervals so as to produce interval judgements corresponding to their temporal perception of the stimuli. In contrast with the primary animals in the experimental group however, the release of $\mathrm{T} 1$ is coupled with no voluntary action. Because the yoked animals continue to experience the T1-T2 association with exactly the same frequency and intervals as the primary animals, any difference in their choice of feeder cannot be explained in terms of their experience of T1's association with T2 alone.

If primary subjects experience temporal binding, they should produce more shortinterval judgments than the yoked subjects when intervals are close to the trained threshold between short and long. In other words, the primary animals would perceive the first tone (T1) as closer to the second cue (T2) more often than the control animals, as a result of the shift forward in time of the executed action. Clearly, interval judgments in the same animal species could vary as a function of the types of stimuli used in the experiment. However, the relevant variables in the experimental setup regard the comparison between the interval judgments during the training and the experimental phase, and between the primary and the yoked animals being subject to different conditions.

The basic experimental proposal that we are making here could be adjusted to test temporal binding in an allocentric perspective. Let's imagine that in this version of the interval judgement test of temporal binding, the yoked animals have the chance to observe the pressing action executed by the primary animals thanks to a transparent panel dividing the two adjacent enclosures. If the yoked animal is able to 1) form an action-effect relation producing a compression in the perception of the interval between the action and the outcome, and 2) if it is able to recognize the same actioneffect relation when the action is executed by another, then the yoked animal would also produce more short-interval judgements than it did during the training phase. (Note that as in the case of social binding in the human case, the magnitude of the temporal compression when the action-effect relation is only observed could be different than when the action is executed by the subject.)

\section{Interpretations of possible experimental outcomes}

In this section we work through the implications of the proposed basic experiment for comparative accounts of causal cognition. The experiment has three (or four, depending how one counts) qualitatively different possible outcomes.

1. (No binding) The timing judgements made by primary and yoked animals show no temporal binding.

2. (Same binding) The timing judgements made by primary and yoked animals both show the temporal binding effect.

3. (Different binding) The temporal binding effect is present in one group, but absent in the other, either (a) present in the primary subjects only or (b) present in the yoked subjects only. 
Findings 1 or 2 would lend support to the view that human processing of causeeffect relations is different from other species. Because different species may have different temporal binding profiles, a finding in any particular animal species should not be generalized to all animals. But if no other species shows temporal binding, finding 1 would be consistent with unique, late-evolved processing of causal relations by humans. Finding 2 would suggest that temporal binding evolved relatively early and could have been later co-opted and specialized for causal processing in humans, or might be a side effect of something else unique to humans. In either case, the distribution of temporal binding among different species is relevant to phylogenetic inferences about the emergence of causal cognition.

A difference of temporal binding between the primary and yoked conditions presents the most interest for understanding causal cognition in animals. We regard finding 3b-temporal binding only in the yoked (non-causal) condition-to be quite unlikely, as it would be a surprising reversal of the pattern in the human case. Finding 3 a would lend support for similar processing of cause-effect relations by humans and animals, and would suggest relatively early evolution of dedicated causal processing.

In addition to the phylogenetic implications of the various outcomes, finding a difference in temporal binding between causal (primary animal) and non-causal (yoked animal) conditions in the basic experiment would have important implications for the debate about the nature of causal representation in animal cognition. Because the stimuli and the actual interval separating them is identical between the paired subjects, if the primary animal forms an association which involves temporal binding and the other animal does not, it shows that the same first-order stimuli can be associated by these animal subjects in two different ways. We refer to the structure relating two stimuli as cause and effect as a structural abstraction because it may be reused for stimuli with different properties. Temporal binding could thus serve as a signature or marker of a structural cause-effect abstraction in nonhuman animals. Structural abstraction of cause-effect relations, if found in nonlinguistic creatures, provides evidence for an intermediate level of representation between first-order associations which merely pair events and symbolic/linguistic representations of cause-effect which provide very rich possibilities for combination and inference.

Finding $3 \mathrm{a}$ also opens up the space for experimental investigation of the conditions in which cause-effect representations are established, and when they are deployed in a variety of cognitive tasks. Some slight modifications of the basic experimental design (already mentioned in the previous section), can be deployed to see whether cause-effect representation in a given species depends on its own agency (as in the primary animal), or whether an observer animal will form such representations upon observing the actions of another (e.g., by modifying our basic experiment to give the yoked animal visual access to the primary animal) or observing the operations of a machine in the role of the primary animal (adapting something like the setup of Buehner, 2012). In the next section we consider in more detail the investigation of abstraction, allocentricity, rationality and explicitness, through such variations of the experimental framework we have sketched so far. 


\section{Approaching intermediate levels of reasoning through psychophysics}

Earlier in the paper, we have described the ongoing dispute in terms of multi-level versus two-level theorists on causal cognition. We have stated that multi-level theorists believe that at least some animals other than humans explicitly reason about causal relations under some conditions. On the contrary, two-level theorists claim that, although nonhuman animals sometimes show differential responses to mere covariation and causation, causal relations are not represented by the animal subject in an explicit and abstract way.

In humans, causal representations are connected to a shift in the perception of the timing of external cues. The relationship between causality and time explored in temporal binding studies with humans suggests that subjects retrieve past events and anticipate future ones through a process that involves restructuring representations of lower-level perceptual features. This restructuring is understood as the reorganization of the internal mechanisms that mediate cue perception: If one cue is represented as causally connected to another cue, then the perception of the former is delayed and the perception of the latter is anticipated, creating an illusory temporal compression between them.

On the basis of these findings, we have proposed to investigate temporal binding experiments in other animal species. A positive finding of the binding effect would provide convergence with the human studies, and suggest novel ways in which representations of causal relations could be inquired into nonverbally. A positive finding of temporal binding in other animal species could also allow the investigation of the features of causal representation (such as explicitness abstraction, allocentricity and rationality) which two-level theorists deny to animals, and which would provide support for an intermediate level of representation, located in-between first-order associations of perceptual features and symbolic processing. The investigation of the intermediate level could be approached through variations of the experimental proposal we have sketched in the earlier section.

\subsection{Explicit representation of cause-effect relation}

Regarding explicitness: In the temporal binding literature, the implicit/explicit distinction is largely used for purposes that are different from our own here. In the context of intentional actions, temporal binding is considered a measure of implicit sense of agency, i.e., the awareness of being the author of an action, without the subject being necessarily cognitively aware of having executed it. Binding is contrasted with judgments (or beliefs) of agency based on verbal reports of action experience.

The later discoveries that binding can occur also without intentional actions have suggested that the shift in time perception in action-effect pairs does not depend on the sensorimotor mechanisms underlying action execution (Desantis \& Buehner, 2019; Desantis et al., 2012). If causal representations are necessary for the binding effect to occur, then the action awareness measured by this phenomenon is not as thin, unconscious and pre-reflective as the expression "implicit sense of agency" could 
suggest (Hoerl et al., 2020; Tramacere, 2021). Because of space limitations, we will not describe the debates on the relationship between awareness of agency and representation of causality here. It will suffice to say that our analysis is based on the interpretation of new evidence that causal representations are necessary (and probably sufficient) for temporal binding. As such, our analysis can indirectly contribute to those debates.

The way the implicit/explicit distinction is typically operationalized in temporal binding studies is not viable in nonlinguistic animals, because they cannot verbally report their own beliefs of agency (or causality), nor can they directly report on the time at which an event occurred. Further, since our goal is to show that temporal binding operationalizes an intermediate level of reasoning that lies in-between first-order representation of perceptual features (usually considered implicit, and at least possibly unconscious) and symbolic reasoning (expressed by verbally reportable beliefs which are by definition explicit, and thus conscious), we must rely on different criteria to identify explicit processes. An intermediate level of (explicit) reasoning could also show dissociations with both lower associative learning processes and higher-levels of representations.

In temporal binding studies, the temporal shift in event perception is present when individuals expect an effect, but it is absent when they only experience targets that are equally predictable because merely associated with a predictor. This suggests that an implicit prediction, i.e., activating the representation of an outcome because associated with a previously experienced cue, is not sufficient for temporal binding to occur in humans. This also supports the proposal to use temporal binding as a measure of the prediction of an effect on the basis of a causal representation.

This interpretation is in line with previous studies in humans (see for example Dale et al., 2012; Vakil et al., 2017) showing that implicit predictions can be understood as the activation of a latent memory trace, which allows the subject to react earlier to a previously experienced association between cues. In contrast, an explicit prediction would consist in actively anticipating the observation of a cue as the result of its assumed relation with another cue. These studies show that implicit predictions are less likely to reach the subject's awareness, while individuals showing explicit anticipation strategies report higher awareness of their behavior. However, the differential contribution of implicit and explicit processes to prediction behavior is still an open question (see Daltrozzo \& Conway, 2014 for a discussion), meaning that without further investigation not too much weight should be placed on the alignment of the studies mentioned at the beginning of this paragraph to the interpretation we are proposing.

Further support for our interpretation comes from recognition that explicit representations can be identified through their potential to impact downstream processing (Shea, 2015). On the account of causal reasoning advanced by Woodward (2011), an explicit causal representation is usually conscious, to some extent available to other systems for inference, reasoning and planning, and integrated with or connected to other knowledge and representation the subject possesses. A similar proposal has been advanced by Papineau (2001). This notion of explicitness is relevant to whether a subject is able to utilize previously formed causal representations to navigate novel situations. 
Thus, if explicit predictions produce time compression between cues, then the prediction of the effect producing binding should be available for further cognitive operations, while mere cue correlation should not have an impact on downstream processing, except via the representations that it connects. In fact, effect prediction producing binding can be used to coordinate joint tasks in social situations (Jenkins et al., 2021; Strother et al., 2010), and to track the positions of stimuli in the context of the oddball effect paradigm (Majchrowicz \& Wierzchon, 2018) in which an unexpected stimulus is inserted into a train of familiar, repetitive stimuli. Further, the prediction of an effect producing a time shift between cues can also provide input for explicit judgements of causality and agency (Antusch et al., 2020; Dogge et al., 2012).

This interpretation would be corroborated if additional studies were conducted which show that temporary impairments of the hippocampus interfere with temporal binding produced by effect anticipation, but not with the capacity to respond to the association between two cues. To date, there are already a number of studies which support the hypothesis that the hippocampus is responsible for retention of stimuli traces, making them available for further processing, but not necessary for basic associative learning (Bangasser et al., 2006; Clark \& Squire, 1998). Clark and Squire (1998) also showed that retention of stimuli traces comes, in humans, with explicit conscious awareness of the stimuli. Therefore, this approach could prove useful for establishing the relationship between temporal binding and explicit awareness.

Note that temporal binding and verbal judgements of causality (or agency) are dissociable; moreover, while temporal binding is more strongly affected by low level features (such as sensitivity to recently experienced cue co-occurrence), verbal judgements of causality are less vulnerable to them (Moore et al., 2012). This again suggests a multi-level view of explicitness (which invites investigations in future studies), with symbolic representations of causality being potentially different from explicit nonsymbolic ones.

In sum, because first-order perceptual accounts of causal behavior involve relatively specific associations between perceptible features of objects that are co-present in situations, they may operate online without the individual needing to activate any explicit expectation on how one event is going to affect the occurrence of a second one. As such, these associations can be steering subjects' behaviors entirely implicitly. On the contrary, an explicit causal representation would support a wider variety of relationships over greater spatial and temporal ranges, allowing the individual to predict the behavior and characteristics of the system, in a way that can be deployed for decision making tasks combining diverse kinds of information in increasingly complex ways.

\subsection{Structural abstraction}

In humans, the binding effect occurs also with unpredictable multiple outcomes. Desantis and colleagues (2012) for example exposed participants to two action effects, such as tones of various intensity, say T2 and T3, associated with a same action cause, say T1. They found that temporal binding was not affected by the identity of the outcome, even if the probability of T2 was lower than the probability of T3. This suggested that temporal binding does not depend on a single-cause-single-effect relation, 
but rather on a nonspecific effect prediction, based on the representation of a generalizable cause-effect relation. Subsequent studies (Haering \& Kiesel, 2014; Haggard et al., 2009) showing that predictive control (the expectation of observing an effect at a specific point in time), more than identity prediction, produces temporal binding. This supports the hypothesis of a generalized template for causal relations because it shows that individuals experiencing binding are more sensitive to the occurrence of an effect than to the actual identity of the effect.

The presence of a representation of a generalizable causal relation could be tested in animal species through the experimental proposal proposed in the previous section. The animal subjects would be trained to discriminate short from long intervals between two tones (T1 and T2) occurring in 75\% of the trials. The subject would then be trained to discriminate short from long intervals between the same action cause (T1) and a different action effect T3, occurring in $25 \%$ of the trials. If the primary animal would show consistent binding ${ }^{2}$ of both tones (T2 and T3) associated with a single cause (the action operationalized through T1), then we could obtain evidence that the subjects have formed a representation of a cause-effect relation which is abstract, because generalizable to different stimuli.

Our use of "abstraction" does not simply refer to the ability to extend object categories to novel instances or generalizing behavioral responses across the members of one category - what we may call respectively "categorical abstraction" and "generalization abstraction". We rather refer to the capacity to dynamically represent the properties of certain objects or events, so that these objects and events are thought to be connected in a particular way to other objects and events. Subjects respond to stimulation in specific ways in virtue of how the properties indicate the specific type of relations. Because the same stimuli may be related in different relational templates, we may call this "structural abstraction".

In line with Piaget's view (Piaget et al., 1951), we think that there is an incremental path from categorical and generalization abstraction to structural abstraction, such that a subject that is capable of the latter must also be capable of the former. In line with Barsalou (2003), we think that the features that are related in an instance of an abstraction only reflect some of the many possible features that can be used to form such abstractions. Once a structural abstraction is constructed for a relation, a trace of it becomes established in memory, increasing the likelihood of re-using the abstraction on another occasion.

Structural abstractions would thus provide a schema for relating the features of at least two variables. Possessing such a schema would in turn dramatically accelerate learning of specific causal, physical or agentive relational properties. Note that structural abstraction also underlies various forms of non-causal cognition such as transitive inference (Allen, 2006) and generalization of the same-different relation.

\footnotetext{
2 It is clearly possible that the magnitude of binding in T1-T2 and T1-T3 trials could change as a function of the perceptual differences between $\mathrm{T} 2$ and $\mathrm{T} 3$, but this confounding factor can be controlled by comparing time judgments in the baseline and experimental conditions.
} 


\subsection{Allocentricity: social perspective}

Temporal binding studies in humans have shown that the observation of another agent executing an action also causes a temporal reduction of action-effect estimates (Pfister et al., 2014; Poonian \& Cunnington, 2013; Strother et al., 2010). As described in the previous section, the basic experimental proposal we have made to test temporal binding in animals could be extended to test binding in observed action-effect relations with them. A positive finding of temporal binding in a social perspective would strengthen the view that temporal binding operationalizes a non-egocentric, thus generalizable cause-effect relation.

This also suggests the possibility of investigating the role of causal representation in other areas of comparative psychology that are contested by two-level and multilevel theorists. Take imitation, for example, which seems to involve mapping the allocentric perspective of the demonstrator onto the egocentric perspective of the observer. In an elegant experiment, Akins and Zentall (1998) tested the behavior of quail after they observed a conspecific demonstrator carry out actions that were either reinforced or not. They found that observers imitated the action only if they also observed the demonstrator being reinforced, a fact which some two-level theorists have attempted to explain in terms of the observers' own first-order reinforcement history. A modification of the experiment could investigate whether imitation rests on observers temporally binding the demonstrators' actions to reward, thus potentially implicating intermediate-level allocentric causal representation in the observers' imitative behavior.

The presence of temporal binding in animal species capable of skilled social cognitive abilities would thus provide evidence for intermediate levels of representation, available for a kind of reasoning where the subjects are not just acting on the basis of previously formed associations of perceptual features with specific responses or outcomes. Rather, they would show explicit, flexible and generalizable understanding of others' behavior, based on their capacity to represent action-effects relations.

\section{Psychophysical perspectives in comparative psychology}

So far, we have elaborated on the possibility of finding temporal binding in animal species, with animals' temporal judgements being tested during the execution of an intentional action or during the observation of an action executed by a peer. Assessing binding in animals' intentional actions would constitute the premises for further psychophysical investigations.

An obvious subsequent step would be testing temporal binding in purely causal non-action conditions, e.g., where the subjects only observe mechanically generated cause-effect relations. The experimental design we have advanced earlier in the paper (Sect. 4) could be adjusted to test binding in purely causal conditions (also called causal binding by Buehner \& Humphreys, 2009). In the experimental phase, one could compare the temporal judgments made by the yoked animals with those made by the primary animals, which now have the chance to observe the lever being pressed by a mechanical device producing the release of $\mathrm{T} 1$ and $\mathrm{T} 2$, separated by variable interval lengths. 
A positive finding of binding in these experiments would provide a first important convergence with human studies, where a shift in time perception has been found in association with causal reasoning. While in principle the convergence of human and nonhuman-animal temporal binding is not a conclusive proof of analogous causal cognitive abilities, it would provide evidence for comparable processes of causal representations affecting perception of event timing.

Findings of temporal binding with intentional actions and possibly with mechanical causes would have implications for the debate between two-level and multi-level theorists about causal cognition, providing support for an intermediate level of causal representation possessed by animals.

Two-level theorists may claim that a positive finding of binding in animal species represents no challenge for their view. They may claim that temporal binding in animals would not prove that animals possess causal beliefs that incorporate a conscious and abstract representation of underlying unobservable mechanisms, such as gravity or weight. A positive finding of binding would only confirm what we already know, namely that some animal species are able to treat causal relations differently from other relations, by reasoning about non-causal factors, such as contingency, contiguity, cue competition, and so on. Alternatively, two-level theorists may claim that the representation operationalized by the temporal binding experiments is not a causal representation, but more modestly an explicit effect anticipation.

We think that while in the animal cognition literature effect anticipation is normally regarded as causal (Blaisdell et al., 2006; Penn \& Povinelli, 2007), it is true that a positive finding of temporal binding would not show that animals employ theoretical concepts to represent causal relations. No theory-like representation is necessary for temporal binding to occur. However, animals showing temporal binding would provide support for an intermediate level of representation, which is disregarded by two-level theorists. If some animal species possess explicit, allocentric and generalizable causal representation, then we cannot reduce all animal behavior to first-order relational reasoning. A multi-level view of animal cognition and consciousness is needed to understand the evolutionary and developmental emergence of human-like causal cognition, as well as the variety and distribution of causal cognition in nonhuman animals (see Clatterbuck, 2018 for an extensive discussion of this point).

Experiments based on temporal binding potentially establish convergent evidence between human and nonhuman causal cognition, even in the absence of a clear functional or adaptive story. Whether the temporal reorganization seen in temporal binding is due to lower-level neural facts (such as how priming works via neural prepotentiation) or because it provides a functional advantage (e.g., by facilitating information processing) is not possible to specify with certainty. Ultimately, our account does not depend on resolving this issue, but it is worth considering current proposals concerning the functional role of temporal binding.

Moore and Obhi (2012) have interpreted experimental results to say that the temporal shift between causes and effects is instrumental to acquiring the direct consequences of intentional behavior. This proposal is supported by a consistent series of studies showing that dopamine modulates the magnitude of temporal binding. If the executed action produces a positive reward-related effect, the temporal shift between the action and the effect is augmented (Aarts et al., 2012). These findings are also consistent 
with research showing that positive rewards facilitate the execution of action control (Aston-Jones \& Cohen, 2005).

It might be too early to draw a conclusion on the adaptive role of temporal binding, especially considering that the action-effect binding may be simply a byproduct of our neurocognitive architecture. Temporal binding might simply be explained by putting it under the range of phenomena which show that beliefs change perception. Regardless of the precise functional significance of temporal binding, the interest that this experimental paradigm raises is that it blocks the premature foreclosure of approaches to the mind of animals and other prelinguistic subjects that attributes more than first-order mechanisms.

Exactly what neural architecture supports causal representation, producing the phenomenon of temporal binding, also remains a matter of theorizing. So far studies have only investigated the neural basis of binding in the context of agency, and identified the activations of a wider number of frontal and parietal areas which integrate visuomotor information during the subject's actions compared to non-action or involuntary conditions (Poonian et al., 2014, for a review see Haggard, 2017). Further, some studies are exploring physiological mechanisms to explain the temporal binding phenomenon. One of these is the acceleration of a pacemaker, i.e., an internal clock that works as an accumulator which would allow estimating time in perceptual tasks. This hypothesis has received experimental support (Fereday et al., 2019).

It is worth saying that the studies showing a wider activation of brain areas during intentional binding tasks match well with Bernard Baars' global workspace theory which provides a unified account of conscious attention, flexible reasoning, and executive control. According to the global workspace theory, information from disparate sources that is otherwise unconscious, and processed only implicitly, can become coupled through the coordinated spread of high levels of activity in frontal and parietal regions (see Baars, 2017), constituting explicit, attentionally focused processing. Baars himself regards global workspace theory as plausibly applied to some nonhuman animals (Boly et al., 2013).

The global workspace approach has its critics, but it is also not the only approach to define implicit processing as unconscious, not accessible to other parts of the cognitive system, and explicit processing as conscious processing available for further operations. Other approaches include dual system theories (e.g., Evans 2003). For our purposes it is not necessary to select among available approaches. Any of them are sufficient to allow space for multi-level positions, by providing a theoretical framework within which temporal binding, and other reportable or actionable aspects of perception, cognition, and consciousness might be understood and further investigated.

A similar suggestion about the value of temporal reorganization of experience for comparative studies involving nonhuman animals is made by Birch et al. (2020) with regard to the "color phi" phenomenon (Kolers \& von Grünau, 1976). In color-phi experiments, two dots of different colors are presented serially with short temporal delay and spatial separation between them. Subjects report this presentation as a single dot moving from the first location to the second location and changing color midway. Since the reported phenomenon locates the color change before the spatio-temporal location of the second dot, this seems to involve a reorganization of experience in 
time that is akin to the temporal binding effect for causal cognition, and potentially studyable without the need for linguistic report.

\section{Conclusion}

In this article, we have proposed that temporal binding can be studied non-linguistically in animals other than humans. In humans, awareness of causal relations is connected to specific changes in perception of event timing, thus providing theoretical reasons for linking temporal binding to explicit causal representations. Therefore, if temporal binding is verified in animals, by theoretically-structured analogy we can attribute explicit causal representation to them.

The finding in animals of temporal binding may restructure the debate between twolevel and multi-level theorists on causal cognition. If proven in animals under a variety of experimental modifications, temporal binding could provide evidence of causal reasoning entailing structurally abstract, explicit and allocentric representations, and providing evidence for rational processes that cannot be explained exclusively through low-level perceptually-linked representations.

Through psychophysics, we can thus understand not only what the subject is perceiving, but also how the subject is perceiving, thus providing a marker of the content of the organism's experience. On the other hand, the meaning of the marker relies on interpretations made during human investigations, and as such it is dependent on interpreting similar experimental outcomes as accounting for approximately similar meanings that are nevertheless revisable for various purposes (Allen, 2014).

Funding No funding.

Availability of data and material Not applicable.

Code availability Not applicable.

\section{Declarations}

Conflict of interest The authors declare that they have no conflicts of interest.

Open Access This article is licensed under a Creative Commons Attribution 4.0 International License, which permits use, sharing, adaptation, distribution and reproduction in any medium or format, as long as you give appropriate credit to the original author(s) and the source, provide a link to the Creative Commons licence, and indicate if changes were made. The images or other third party material in this article are included in the article's Creative Commons licence, unless indicated otherwise in a credit line to the material. If material is not included in the article's Creative Commons licence and your intended use is not permitted by statutory regulation or exceeds the permitted use, you will need to obtain permission directly from the copyright holder. To view a copy of this licence, visit http://creativecommons.org/licenses/ by $/ 4.0 \%$. 


\section{References}

Aarts, H., Bijleveld, E., Custers, R., Dogge, M., Deelder, M., Schutter, D., \& van Haren, N. E. M. (2012). Positive priming and intentional binding: Eye-blink rate predicts reward information effects on the sense of agency. Social Neuroscience, 7(1), 105-112. https://doi.org/10.1080/17470919.2011.590602

Akins, C. K., \& Zentall, T. R. (1998). Imitation in Japanese quail: The role of reinforcement of demonstrator responding. Psychonomic Bulletin \& Review, 5(4), 694-697. https://doi.org/10.3758/BF03208847

Allen, C. (2006). Transitive inference in animals: Reasoning or conditioned associations? In S. Hurley \& M. Nudds (Eds.), Rational animals? (pp. 175-185). Oxford University Press. https://doi.org/10.1093/ acprof:oso/9780198528272.003.0007

Allen, C. (2014). Umwelt or Umwelten? How should shared representation be understood given such diversity? Dimensions of Zoosemiotics, 198(1-4), 137-158. https://doi.org/10.1515/sem-2013-0105

Antusch, S., Custers, R., Marien, H., \& Aarts, H. (2020). Intentionality and temporal binding: Do causality beliefs increase the perceived temporal attraction between events? Consciousness and Cognition, 77, 102835. https://doi.org/10.1016/j.concog.2019.102835

Aston-Jones, G., \& Cohen, J. D. (2005). An integrative theory of locus coeruleus-norepinephrine function: Adaptive gain and optimal performance. Annual Review of Neuroscience, 28(1), 403-450. https://doi. org/10.1146/annurev.neuro.28.061604.135709

Baars, B. J. (2017). The global workspace theory of consciousness. In S. Schneider \& M. Velmans (Eds.), The Blackwell companion to consciousness (pp. 227-242). Wiley. https://doi.org/10.1002/ 9781119132363.ch16

Bangasser, D. A., Waxler, D. E., Santollo, J., \& Shors, T. J. (2006). Trace conditioning and the hippocampus: The importance of contiguity. The Journal of Neuroscience: The Official Journal of the Society for Neuroscience, 26(34), 8702-8706. https://doi.org/10.1523/JNEUROSCI.1742-06.2006

Barsalou, L. W. (2003). Abstraction in perceptual symbol systems. Philosophical Transactions of the Royal Society of London Series B: Biological Sciences, 358(1435), 1177-1187. https://doi.org/10.1098/rstb. 2003.1319

Birch, J., Schnell, A. K., \& Clayton, N. S. (2020). Dimensions of Animal Consciousness. Trends in Cognitive Sciences, 24(10), 789-801. https://doi.org/10.1016/j.tics.2020.07.007

Blaisdell, A. P., Sawa, K., Leising, K. J., \& Waldmann, M. R. (2006). Causal reasoning in rats. Science (new York, NY), 311(5763), 1020-1022. https://doi.org/10.1126/science.1121872

Blakey, E., Tecwyn, E. C., McCormack, T., Lagnado, D. A., Hoerl, C., Lorimer, S., \& Buehner, M. J. (2019). When causality shapes the experience of time: Evidence for temporal binding in young children. Developmental Science, 22(3), e12769. https://doi.org/10.1111/desc.12769

Boly, M., Seth, A. K., Wilke, M., Ingmundson, P., Baars, B., Laureys, S., Edelman, D., \& Tsuchiya, N. (2013). Consciousness in humans and non-human animals: Recent advances and future directions. Frontiers in Psychology. https://doi.org/10.3389/fpsyg.2013.00625

Buckner, C. (2011). Two Approaches to the Distinction between Cognition and 'Mere Association.' International Journal of Comparative Psychology, 24(4). https://escholarship.org/uc/item/1x28x459

Buehner, M. J. (2012). Understanding the past, predicting the future: Causation, not intentional action, is the root of temporal binding. Psychological Science, 23(12), 1490-1497. https://doi.org/10.1177/ 0956797612444612

Buehner, M. J. (2015). Awareness of voluntary and involuntary causal actions and their outcomes. Psychology of Consciousness: Theory, Research, and Practice, 2(3), 237-252. https://doi.org/10.1037/ cns0000068

Buehner, M. J., \& Humphreys, G. R. (2009). Causal Binding of Actions to Their Effects. Psychological Science, 20(10), 1221-1228. https://doi.org/10.1111/j.1467-9280.2009.02435.x

Civelek, Z., Call, J., \& Seed, A. M. (2020). Inferring unseen causes: Developmental and evolutionary origins. Frontiers in Psychology. https://doi.org/10.3389/fpsyg.2020.00872

Clark, R. E., \& Squire, L. R. (1998). Classical conditioning and brain systems: The role of awareness. Science, 280(5360), 77-81. https://doi.org/10.1126/science.280.5360.77

Clatterbuck, H. (2018). The Logical Problem and the Theoretician's Dilemma. Philosophy and Phenomenological Research, 97(2), 322-350. https://doi.org/10.1111/phpr.12331

Cravo, A. M., Claessens, P. M. E., \& Baldo, M. V. C. (2009). Voluntary action and causality in temporal binding. Experimental Brain Research, 199(1), 95-99. https://doi.org/10.1007/s00221-009-1969-0 
Dale, R., Duran, N. D., \& Morehead, J. R. (2012). Prediction during statistical learning, and implications for the implicit/explicit divide. Advances in Cognitive Psychology, 8(2), 196-209. https://doi.org/10. 2478/v10053-008-0115-z

Daltrozzo, J., \& Conway, C. M. (2014). Neurocognitive mechanisms of statistical-sequential learning: What do event-related potentials tell us? Frontiers in Human Neuroscience. https://doi.org/10.3389/fnhum. 2014.00437

Desantis, A., \& Buehner, M. (2019). Causality guides time perception. In V. Arstila, A. Bardon, S. E. Power, \& A. Vatakis (Eds.), The illusions of time: philosophical and psychological essays on timing and time perception (pp. 187-203). Springer. https://doi.org/10.1007/978-3-030-22048-8_11

Desantis, A., Hughes, G., \& Waszak, F. (2012). Intentional binding is driven by the mere presence of an action and not by motor prediction. PLOS ONE, 7(1), e29557. https://doi.org/10.1371/journal.pone. 0029557

Dogge, M., Schaap, M., Custers, R., Wegner, D. M., \& Aarts, H. (2012). When moving without volition: Implied self-causation enhances binding strength between involuntary actions and effects. Consciousness and Cognition, 21(1), 501-506. https://doi.org/10.1016/j.concog.2011.10.014

Eagleman, D. M., \& Holcombe, A. O. (2002). Causality and the perception of time. Trends in Cognitive Sciences, 6(8), 323-325. https://doi.org/10.1016/S1364-6613(02)01945-9

Engbert, K., Wohlschläger, A., Thomas, R., \& Haggard, P. (2007). Agency, subjective time, and other minds. Journal of Experimental Psychology: Human Perception and Performance, 33(6), 1261-1268. https:// doi.org/10.1037/0096-1523.33.6.1261

Evans, J. S. B. T. (2003). In two minds: Dual-process accounts of reasoning. Trends in Cognitive Sciences, 7(10), 454-459. https://doi.org/10.1016/j.tics.2003.08.012

Fereday, R., Buehner, M. J., \& Rushton, S. K. (2019). The role of time perception in temporal binding: Impaired temporal resolution in causal sequences. Cognition, 193, 104005. https://doi.org/10.1016/j. cognition.2019.06.017

Gopnik, A., Glymour, C., Sobel, D. M., Schulz, L. E., Kushnir, T., \& Danks, D. (2004). A theory of causal learning in children: Causal maps and Bayes nets. Psychological Review, 111, 3-32. https://doi.org/ 10.1037/0033-295X.111.1.3

Haering, C., \& Kiesel, A. (2014). Intentional Binding is independent of the validity of the action effect's identity. Acta Psychologica, 152, 109-119. https://doi.org/10.1016/j.actpsy.2014.07.015

Haggard, P. (2017). Sense of agency in the human brain. Nature Reviews Neuroscience, 18(4), 196-207. https://doi.org/10.1038/nrn.2017.14

Haggard, P., Clark, S., \& Kalogeras, J. (2002). Voluntary action and conscious awareness. Nature Neuroscience, 5(4), 382-385. https://doi.org/10.1038/nn827

Haggard, P., Poonian, S., \& Walsh, E. (2009). Representing the consequences of intentionally inhibited actions. Brain Research, 1286, 106-113. https://doi.org/10.1016/j.brainres.2009.06.020

Heyes, C. (2018a). Cognitive gadgets: The cultural evolution of thinking (p. 292). Harvard University Press. https://doi.org/10.4159/9780674985155

Heyes, C. (2018b). Enquire within: Cultural evolution and cognitive science. Philosophical Transactions of the Royal Society of London Series b, Biological Sciences, 373(1743), 20170051. https://doi.org/ 10.1098/rstb.2017.0051

Hoerl, C., Lorimer, S., McCormack, T., Lagnado, D. A., Blakey, E., Tecwyn, E. C., \& Buehner, M. J. (2020). Temporal binding, causation, and agency: Developing a new theoretical framework. Cognitive Science, 44(5), e12843. https://doi.org/10.1111/cogs.12843

Jacobs, I. F., \& Osvath, M. (2015). The string-pulling paradigm in comparative psychology. Journal of Comparative Psychology, 129(2), 89-120. https://doi.org/10.1037/a0038746

Jenkins, M., Esemezie, O., Lee, V., Mensingh, M., Nagales, K., \& Obhi, S. S. (2021). An investigation of "We" agency in co-operative joint actions. Psychological Research Psychologische Forschung. https:// doi.org/10.1007/s00426-020-01462-6

Kolers, P. A., \& von Grünau, M. (1976). Shape and color in apparent motion. Vision Research, 16(4), 329-335. https://doi.org/10.1016/0042-6989(76)90192-9

Legaspi, R., \& Toyoizumi, T. (2019). A Bayesian psychophysics model of sense of agency. Nature Communications, 10(1), 4250. https://doi.org/10.1038/s41467-019-12170-0

Majchrowicz, B., \& Wierzchoń, M. (2018). Unexpected action outcomes produce enhanced temporal binding but diminished judgement of agency. Consciousness and Cognition, 65, 310-324. https://doi.org/ 10.1016/j.concog.2018.09.007 
McCormack, T., Hoerl, C., \& Butterfill, S. (2011). Tool Use and Causal Cognition. Oxford University Press, Oxford. https://doi.org/10.1093/acprof:oso/9780199571154.003.0001

Moore, J., \& Haggard, P. (2008). Awareness of action: Inference and prediction. Consciousness and Cognition, 17(1), 136-144. https://doi.org/10.1016/j.concog.2006.12.004

Moore, J. W., Lagnado, D., Deal, D. C., \& Haggard, P. (2009). Feelings of control: Contingency determines experience of action. Cognition, 110(2), 279-283. https://doi.org/10.1016/j.cognition.2008.11.006

Moore, J. W., Middleton, D., Haggard, P., \& Fletcher, P. C. (2012). Exploring implicit and explicit aspects of sense of agency. Consciousness and Cognition, 21(4), 1748-1753. https://doi.org/10.1016/j.concog. 2012.10.005

Moore, J. W., \& Obhi, S. S. (2012). Intentional binding and the sense of agency: A review. Consciousness and Cognition, 21(1), 546-561. https://doi.org/10.1016/j.concog.2011.12.002

Nolden, S., Haering, C., \& Kiesel, A. (2012). Assessing intentional binding with the method of constant stimuli. Consciousness and Cognition, 21(3), 1176-1185. https://doi.org/10.1016/j.concog.2012.05.003

Papineau, D. (2001). The evolution of means-end cognition; Why animals cannot think. Royal Institute of Philosophy Supplements, 49, 145-178. https://doi.org/10.1017/S1358246100007153

Papineau, D., \& Heyes, C. (2006). Rational or associative: Imitation in Japanese Quail. In S. Hurley \& M. Nudds (Eds.), Rational animals? (pp. 187-195). Oxford University Press. https://doi.org/10.1093/ acprof:oso/9780198528272.003.0008

Penn, D. C., \& Povinelli, D. J. (2007). Causal cognition in human and nonhuman animals: A comparative, critical review. Annual Review of Psychology, 58(1), 97-118. https://doi.org/10.1146/annurev.psych. 58.110405 .085555

Penn, D. C., \& Povinelli, D. J. (2009). On becoming approximately rational: The relational reinterpretation hypothesis. In S. Watanabe, L. Huber, A. Blaisdel, \& A. Young (Eds.), Rational animals, irrational humans (p. 21). Tokyo: Keio University Press.

Pfister, R., Obhi, S. S., Rieger, M., \& Wenke, D. (2014). Action and perception in social contexts: Intentional binding for social action effects. Frontiers in Human Neuroscience. https://doi.org/10.3389/fnhum. 2014.00667

Piaget, J. (1954). The construction of reality in the child. Routledge. https://www.apa.org/pubs/books/ 4320469

Piaget, J., Piercy, M., \& Berlyne, D. E. (1951). The psychology of intelligence. Philosophical Quarterly, 1(5), 470-471. https://doi.org/10.2307/2216329

Poonian, S. K., \& Cunnington, R. (2013). Intentional binding in self-made and observed actions. Experimental Brain Research, 229(3), 419-427. https://doi.org/10.1007/s00221-013-3505-5

Poonian, S. K., McFadyen, J., Ogden, J., \& Cunnington, R. (2014). Implicit agency in observed actions: Evidence for N1 suppression of tones caused by self-made and observed actions. Journal of Cognitive Neuroscience, 27(4), 752-764. https://doi.org/10.1162/jocn_a_00745

Povinelli, D. J., \& Henley, T. (2020). More rope tricks reveal why more task variants will never lead to strong inferences about higher-order causal reasoning in chimpanzees. Animal Behavior and Cognition, 7(3), 392-418. https://doi.org/10.26451/abc.07.03.08.2020

Povinelli, D. J., \& Penn, D. C. (2011). Through a floppy tool darkly. In T. McCormack, C. Hoerl, \& S. Butterfill (Eds.), Tool use and causal cognition (pp. 69-88). Oxford University Press. https://doi.org/ 10.1093/acprof:oso/9780199571154.003.0004

Ruess, M., Thomaschke, R., \& Kiesel, A. (2017). The time course of intentional binding. Attention, Perception, \& Psychophysics, 79(4), 1123-1131. https://doi.org/10.3758/s13414-017-1292-y

Seed, A., Hanus, D., \& Call, J. (2011). Causal knowledge in Corvids, primates and children: More than meets the eye? Tool Use and Causal Cognition. https://doi.org/10.1093/acprof:oso/9780199571154. 003.0005

Shea, N. (2015). Distinguishing top-down from bottom-up effects. In S. Biggs, M. Matthen, \& D. Stokes (Eds.), Perception and its modalities (pp. 73-91). Oxford: Oxford University Press. https://doi.org/10. 1093/acprof:oso/9780199832798.001.0001

Starzak, T. B., \& Gray, R. D. (2021). Towards ending the animal cognition war: A three-dimensional model of causal cognition. Biology and Philosophy, 36(2), 1-24. https://doi.org/10.1007/s10539-02109779-1

Strother, L., House, K. A., \& Obhi, S. S. (2010). Subjective agency and awareness of shared actions. Consciousness and Cognition, 19(1), 12-20. https://doi.org/10.1016/j.concog.2009.12.007

Suzuki, K., Lush, P., Seth, A. K., \& Roseboom, W. (2019). Intentional binding without intentional action. Psychological Science, 30(6), 842-853. https://doi.org/10.1177/0956797619842191 
Taylor, A. H., Elliffe, D., Hunt, G. R., \& Gray, R. D. (2010). Complex cognition and behavioural innovation in New Caledonian crows. Proceedings of the Royal Society B: Biological Sciences, 277(1694), 2637-2643. https://doi.org/10.1098/rspb.2010.0285

Taylor, A. H., Hunt, G. R., Holzhaider, J. C., \& Gray, R. D. (2007). Spontaneous metatool use by new Caledonian crows. Current Biology, 17(17), 1504-1507. https://doi.org/10.1016/j.cub.2007.07.057

Taylor, A. H., Miller, R., \& Gray, R. D. (2012). New Caledonian crows reason about hidden causal agents. Proceedings of the National Academy of Sciences of the United States of America, 109(40), $16389-16391$.

Tramacere, A. (2021). A causal view of subjective agency. Philosophical Psychology, 2021, 1-24.

Vakil, E., Bloch, A., \& Cohen, H. (2017). Anticipation measures of sequence learning: Manual versus oculomotor versions of the serial reaction time task. The Quarterly Journal of Experimental Psychology, 70(3), 579-589. https://doi.org/10.1080/17470218.2016.1172095

Wen, W., Yamashita, A., \& Asama, H. (2015). The influence of action-outcome delay and arousal on sense of agency and the intentional binding effect. Consciousness and Cognition, 36, 87-95. https://doi.org/ 10.1016/j.concog.2015.06.004

Woodward, J. (2011). A philosopher looks at tool use and causal understanding. In T. McCormack, C. Hoerl, \& S. Butterfill (Eds.), Tool use and causal cognition. Oxford University Press. https://doi.org/ 10.1093/acprof:oso/9780199571154.003.0002

Woodward, J. (2021). Causation with a human face: Normative theory and descriptive psychology. Oxford University Press.

Publisher's Note Springer Nature remains neutral with regard to jurisdictional claims in published maps and institutional affiliations. 